\title{
Constraints on Parton Density Functions from DØ
}

\author{
Jonathan Hays for the D $\varnothing$ Collaboration \\ Imperial College London - Department Of Physics \\ London SW7 2AZ - United Kingdom
}

Five recent results from $D \varnothing$ which either impact or have the potential to impact on uncertainties in parton density functions are presented.

\section{Introduction}

Many analyses at $\mathrm{D} \varnothing$ are sensitive to the modelling of the partonic structure of the proton. When theoretical and experimental uncertainties are well controlled there exists the possibility for additional constraints on parton density functions (PDF). Five measurements are presented which either have already been included in global parton fits or have the potential to contribute in the future.

\section{$2 \quad$ Z Boson Rapidity Distribution}

$\mathrm{D} \emptyset$ measures the differential cross-section divided by the total $\mathrm{Z}$ cross section[2] using $0.4 \mathrm{fb}^{-1}$ of data in the electron decay channel. Isolated electrons are selected with a pseudorapidity of $|\eta|^{\mathrm{a}}<0.9$ or $1.5<|\eta|<$ 3.2. The (next-to) leading electron is required to have a reconstructed transverse momentum, $p_{T}>(15) 25 \mathrm{GeV}$. The dielectron invariant mass must be close to the $\mathrm{Z}$ pole, $71<M_{e e}<111 \mathrm{GeV}$. The accessible $\mathrm{Z}$ rapidities, $|y|<3$, allow probing of quark momentum fractions, $x$, roughly in the range $0.003<x<0.8$. Backgrounds typically arise at between $<1 \%$ to $5 \%$ from multi-jet and electron plus jet processes where one or more jets fake an electron. The dominant sources of systematic uncertainties come from the electron efficiencies and background modelling for small rapidities and PDF uncertainties for

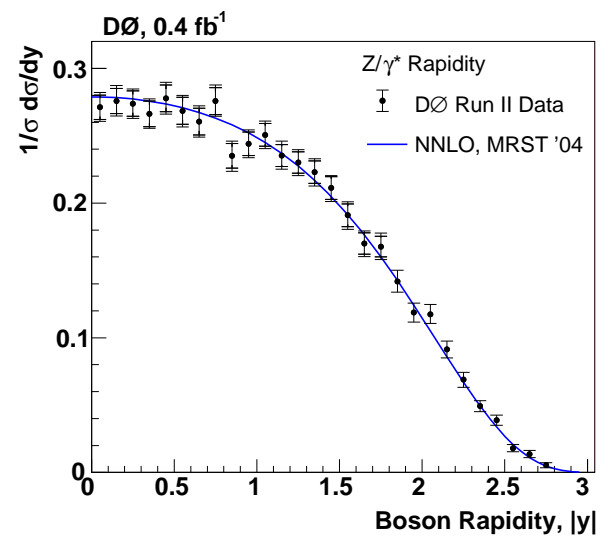

Figure 1: Z Boson rapidity. Inner (outer) error bars indicate statistical (total) uncertainty. A NNLO[3] calculation using MRST[7] 2004 PDFs is shown as a solid line.

the higher rapidities. The result can be seen in Figure 1 as a function of rapidity and is statistics limited. Theoretical predictions at $\mathrm{NNLO}[3]$ are in good agreement with the data.

\section{W Charge Asymmetry}

\footnotetext{
${ }^{\mathrm{a}} \eta=-\ln [\tan (\theta / 2)]$, where $\theta$ is the polar angle
} 


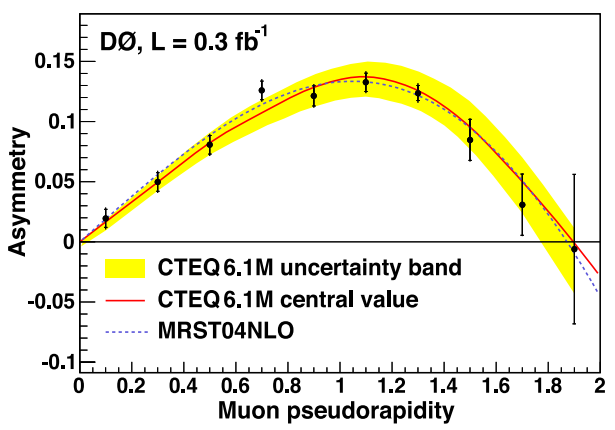

Figure 2: Charge asymmetry versus muon pseudorapidity. Solid line shows a NLO calculation[5] using CTEQ6.1M[6]. The dashed line shows theoretical prediction using MRST04[7] NLO PDFs
Differences in the $u$ and $d$ quark PDFs lead to a charge asymmetry in the $\mathrm{W}$ boson rapidity distribution. $\mathrm{D} \varnothing$ measures the W charge asymmetry in the muon channel, which is sensitive to the ratio of the $u$ and $d$ quark PDFs, using $0.3 \mathrm{fb}^{-1}$ of data[4]. The neutrino in the final state makes the reconstruction of the boson rapidity difficult so the lepton asymmetry is measured, introducing an additional effect from the well understood (V-A) decay. Muons are required to be isolated with $p_{T}>20 \mathrm{GeV}$ and $|\eta|<2$ and the event must have missing transverse energy, $\mathbb{E}_{T}>20 \mathrm{GeV}$ and transverse mass $M_{T}>40 \mathrm{GeV}$. Main backgrounds at the level of a few percent come from electroweak processes such as: $\mathrm{Z} \rightarrow \mu \mu$ and $\mathrm{W} \rightarrow \tau \nu$ and multijet events.

The result is statistics limited and is shown in Figure 2. The theoretical prediction is shown as a solid line and demonstrates reasonable agreement with the data.

\section{Inclusive Jet Production}

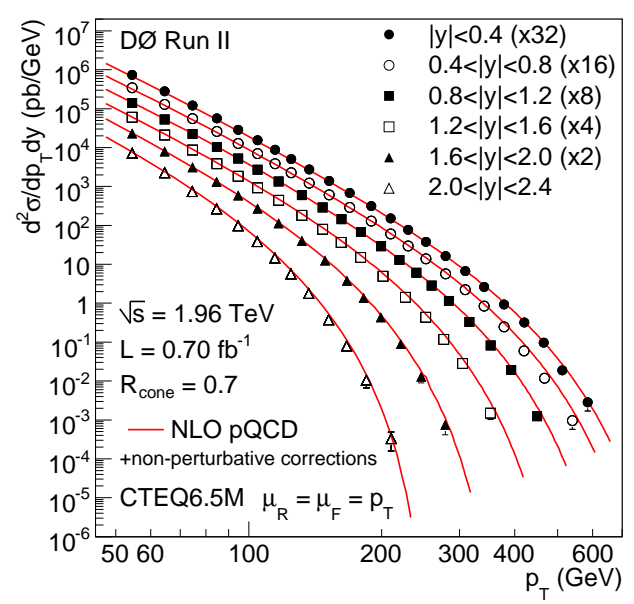

Figure 3: The inclusive jet cross section as a function of jet $p_{T}$ in six $|y|$ bins.

FASTNLO [9] program and CTEQ6.5M[6] PDFs. Additional corrections for nonperturbative effects are applied. Experimental systematic uncertainties are smaller than the PDF uncertainties and in most bins of the same order as the theoretical uncertainties (estimated by varying renormalisation and factorization scales). In all regions the predictions lows for stringent tests of QCD. For high jet $p_{T} \mathrm{~s}$, utilising a wide acceptance in rapidity, the cross section is directly sensitive to the PDFs of the proton. DØ measure the double differential cross section using $0.7 \mathrm{fb}^{-1}$ of data[8] as a function of $p_{T}$, from $p_{T}>$ $50 \mathrm{GeV}$, in six bins in rapidity, extending out to $|y|<2.4$. Jets are reconstructed using a seed-based mid-point cone algorithm with a radius $\mathcal{R}=\sqrt{(\Delta y)^{2}+(\Delta \phi)^{2}}=$ 0.7. Jet corrections are applied to take into account the effects of calorimeter response, out-of-cone showering, pile-up and multiple interactions. Further corrections are applied due to the differing response to quark and gluon initiated jets. Figure 3 shows the results in six bins of rapidity, with perturbative QCD predictions at NLO in $\alpha_{S}$ derived using the
PDFs. Additional corrections for non-

Measuring the inclusive jet cross section al-

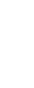

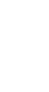

(1)

DIS 2008 
agree with the data.

\section{Photon + Jet Production}

The measurement of the production of a photon associated with jets allows for a powerful probe of QCD. Isolated final state photons in $p \bar{p} \rightarrow \gamma+$ jet $+X$ events are expected to arise dominantly through Compton-like $q g \rightarrow q \gamma$ and annihilation $q \bar{q} \rightarrow g \gamma$ processes. In particular the Compton process dominates at low $p_{T}^{\gamma}<120 \mathrm{GeV}$. $\mathrm{D} \emptyset$ uses $1 \mathrm{fb}^{-1}$ of data to measure the differential cross section versus $p_{T}^{\gamma}$ in four bins representing different angular configurations of the jets and photon[10]. Isolated photons are selected with $\left|y^{\gamma}\right|<1$ and $30<p_{T}^{\gamma}<400 \mathrm{GeV}$. The leading jet is required to be either central $\left|y^{\text {jet }}\right|<0.8$ or forward $1.5<\left|y^{\text {jet }}\right|<2.5$ and with $p_{T}^{\text {jet }}>15 \mathrm{GeV}$. The four angular configurations studied are: $y^{\gamma} y^{\text {jet }}<0, y^{\gamma} y^{\text {jet }}>0$ each for forward and for central jets. The measurement covers a kinematic range in $x$ and $Q^{2}$ of $0.007<x<0.8$ and $900<$ $Q^{2}<1.6 \times 10^{5} \mathrm{GeV}^{2}$ and in particular at low- $x$ is sensitive to the gluon PDF. Photonlike backgrounds from $\pi^{0}, \eta$ etc. and from misidentified jets are suppressed using neu-

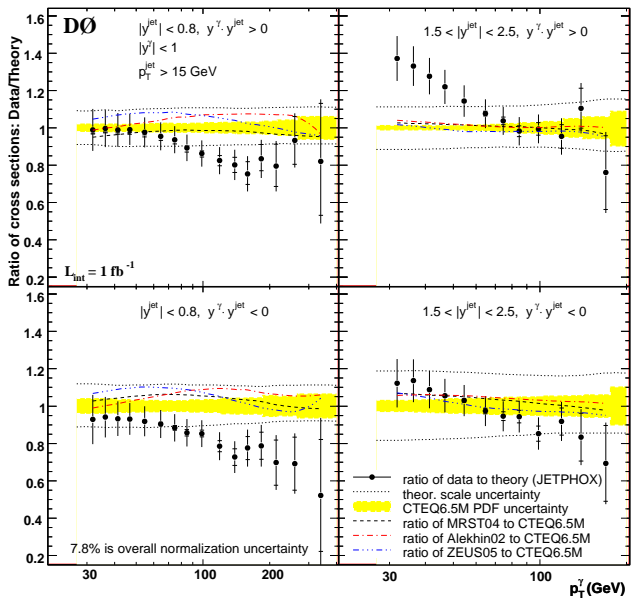

Figure 4: The ratio of the measured differential cross section, in each interval, to the NLO QCD prediction. The dotted lines represent the effect of a factor of two variation in the theoretical scales. A common $7.8 \%$ normalisation uncertainty is not shown on the data points.

ral networks. A sliding cut on $\mathbb{E}_{T}$ is used

to reject backgrounds from cosmic rays and $W \rightarrow e \nu$ events. Statistical uncertainties vary from $0.2 \%$ to $20 \%$ for the most and least populated bins respectively. The experimental systematics contribute around $10 \%$ to $15 \%$ to the overall experimental uncertainty. Figure 4 shows the ratio of the measured cross section to theory calculations at NLO using JETPHOX[11, 12] and CTEQ6.5M[6] PDFs and BFG[13] fragmentation for the four measurement bins. The NLO predictions using a variety $[6,7,14,15]$ of PDFs are not able to simultaneously describe the shape of the $p_{T}^{\gamma}$ dependence of the cross section across the whole range. Futhermore, theoretical scale variation cannot simultaneously describe the data-totheory ratio in each of the four regions. This points to a need for an improved description of $\gamma+$ jet production.

\section{$6 \mathrm{~W}+$ charm production}

The CKM suppression of the leading order $d$-quark-gluon fusion process means $\mathrm{W}+$ charm production provides direct sensitivity to the strange quark $\mathrm{PDF}$ at high $Q^{2}$. D $\varnothing$ measures the ratio of the cross sections: $\sigma(p \bar{p} \rightarrow \mathrm{W}+c-$ jet $) / \sigma(p \bar{p} \rightarrow \mathrm{W}+$ jets $)$ using $1 \mathrm{fb}^{-1}$ of data[16]. 
This ratio allows the cancellation of a number of experimental and theoretical uncertainties. W bosons are selected using their decays to electrons and muons and requiring large $\mathbb{E}_{T}$ in the event. Charm candidates are identified by the presence of muons within a reconstructed jet and making use of the charge correlation between the muon from the charm decay and the lepton from the $\mathrm{W}$ decay. The measured fraction of $\mathrm{W}+$ charm in the $\mathrm{w}+$ jets sample is $0.074 \pm 0.019_{\text {stat }}+0.012-0.014_{\text {syst }}$. This agrees with theoretical predictions and corresponds to a $3.5 \sigma$ significant signal.

\section{Summary}

Five analyses have been presented which are sensitive to the proton PDFs. The first three have already been included in some global parton fits. The analyses presented have only made use of up to around $1 \mathrm{fb}^{-1}$ of data whereas around $4 \mathrm{fb}^{-1}$ are (at the time of writing) recorded on tape. Once theoretical understanding has improved and additional data has been analysed the final two analyses could also provide important inputs to these fits.

\section{References}

[1] Slides: http: //indico. cern. ch/contributionDisplay . py? contribId=99\&sessionId=17\&conf Id=24657

[2] V. M. Abazov et al., Phys. Rev. D 76, 012003 (2007).

[3] C. Anastansiou, L. Dixon, K. Melnikov and F. Petriello, Phys. Rev. D 69, 094008 (2004).

[4] V. M. Abazov et al., Phys. Rev. D 77, 011106R (2008).

[5] C. Balazs and C. -P. Yuan, Comput. Phys. Commun. 79, 291 (1994).

[6] W. K.Tung et al., JHEP 0702, 053 (2007); J. Pumplin et al., JHEP 0207, 12 (2002), D. Stump et al., JHEP 0310, 046 (2003).

[7] A. D. Martin, R. G. Roberts, W. J. Sitrling, and R. S. Thorne, Phys. Lett. B 604, 61 (2004).

[8] V. M. Abazov et al., arXiv:hep-ex/0802.2400 (Accepted by Phys. Rev. Lett.)

[9] T. Kluge, K. Rabbertz, M. Wobisch, arXiv:hep-ph/0609285.

[10] V. M. Abazov et al., arXiv:hep-ex/0804.1107 (Submitted to Phys. Lett. B).

[11] P. Aurenche et al.,Nucl. Phys. B297, 661 (1988); F. Aversa et al., Nucl. Phys. B327, 105 (1989).

[12] S. Catani et al., JHEP 05, 028 (2002).

[13] L. Bourhis, M. Fontannaz, and J. P. Guillet, Eur. Phys. J. C 2, 529 (1998).

[14] S. Alekhin et al., Phys. Rev. D 68, 014002 (2003).

[15] S.Chekanov et al. (ZEUS Collaboration), Eur. Phys. J. C 42, 1 (2005).

[16] V. M. Abazov et al., Phys. Lett. B 666, 23 (2008). 